\title{
Study on the Fundamental Characteristics of the $\Theta$ Probe and Crack Monitoring Using This Probe Combined with the Caustic Method*
}

\author{
Kouichi SEKINO $^{* *}$, Koji SHIMIZU $^{* * *}$ and Takashi FUJIMOTO ${ }^{* * * *}$ \\ ${ }^{* *}$ Graduate School of Engineering, Kanto Gakuin University, \\ 1-50-1, Mutsuura-higashi, Kanazawa-ku, Yokohama 236-8501, Japan \\ E-mail: sekino-kouichi@japeic.co.jp \\ *** Kanto Gakuin University, \\ 1-50-1, Mutsuura-higashi, Kanazawa-ku, Yokohama 236-8501, Japan \\ **** Marktec Corp., \\ 4-17-35, Oomori-nishi, oota-ku, Tokyo 143-0015, Japan
}

\begin{abstract}
The new $\Theta$ probe, which is used in eddy current testing, has various advantages compared to previous probes. In the present study, the fundamental characteristics of this probe are first investigated using commercially available aluminum plates. The effects of liftoff and the angle between the detecting coil and the flaw on the flaw signal are then clarified. The effects of the flaw dimensions on the flaw signal are also investigated, and it is shown that the amplitude reaches a constant value for a flaw depth of $H>8 \mathrm{~mm}$, a flaw width of $W>7 \mathrm{~mm}$, and a flaw length of $L>20$ $\mathrm{mm}$, within the experimental conditions of the present study. Next, the monitoring of crack initiation under static loading and crack growth under the low cycle fatigue condition in the duralumin plates is attempted by applying the $\Theta$ probe combined with the caustic method. It is shown that the signal amplitude can be obtained when fracture is initiated under static loading. In addition, in the A7075 plate, the amplitude is increased at the fatigue crack growth rate of $d a / d N$ larger than 0.017 $\mathrm{mm} / \mathrm{cycle}$. Consequently, the possibility of crack monitoring using the $\Theta$ probe is confirmed.
\end{abstract}

Key words: Nondestructive Inspection, Eddy Current Test, $\Theta$ Probe, Caustic Method, Crack, Stress Intensity Factor, Fracture, Fatigue

\section{Introduction}

The detection of cracks in various machine structures, such as atomic power plants and airplanes, is critical in order to prevent fracture. The eddy current testing method is one technique that has been proposed for the detection of such cracks. However, the detection accuracy of cracks in the usual surface coil by eddy current testing method is rather low ${ }^{(1)}$, because the change in liftoff, the distance between the specimen and the probe, influences the signal amplitude of the surface coil.

Recently, a new eddy current probe with minimal liftoff noise, called the $\Theta$ probe, was developed by Hoshikawa et al. ${ }^{(1)(2)}$. A number of studies ${ }^{(1)-(3)}$ have examined the fundamental characteristics of the eddy current testing using the $\Theta$ probe with respect to phase angle analysis and the influence of the small flaw to the flaw signal. In addition, the effect of liftoff on the phase angle has been investigated using brass plates ${ }^{(2)}$. In contrast, crack monitoring is studied using the eddy current testing method. Fukutomi et al. investigated crack detection in the land-based gas turbine blades using a conventional surface coil ${ }^{(4)}$. Kasuya et al. studied the method of quantitative crack monitoring based on 
the in-situ Eddy Current Monitoring system and revealed that it is possible to monitor quantitatively the crack growth process by this system ${ }^{(5)}$. However, there has been no study on the application of the $\Theta$ probe for monitoring crack initiation.

In the present paper, the effects of liftoff and flaw dimensions on the flaw signal are investigated experimentally in detail using commercially available aluminum plates. In addition, the monitoring of crack initiation and crack growth in duralumin plates under static and low cycle fatigue conditions is attempted by applying the $\Theta$ probe combined with the caustic method (6)(7). The relationship between the flaw signal of the $\Theta$ probe and fracture is investigated by observing the deformation in the vicinity of the crack tip by the caustic method.

\section{Nomenclature}

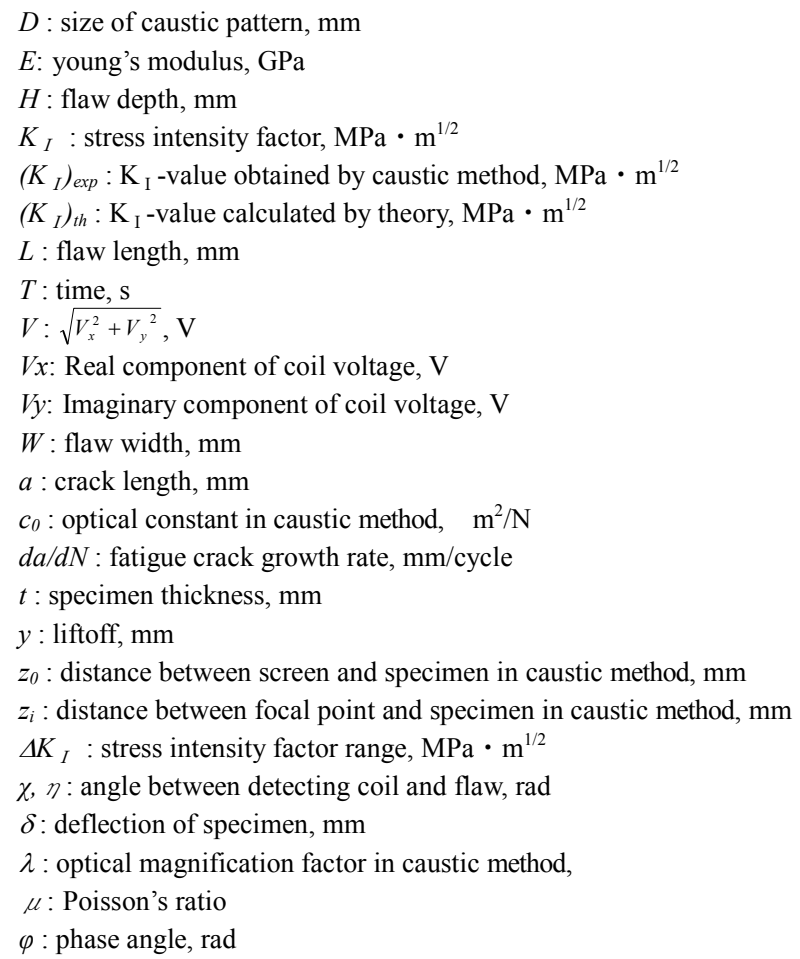

\section{Principle of the Experimental Technique}

\subsection{Eddy Current Test ${ }^{(1)(2)}$}

The $\Theta$ probe consists of a detecting tangential coil and an exciting pancake coil, as shown in Fig. 1. The eddy current is generated in the specimen when an alternating current is given to the exciting coil and a reacted magnetic flux arises due to this eddy current. The voltage of the detecting coil does not change when there is no flaw in the specimen.

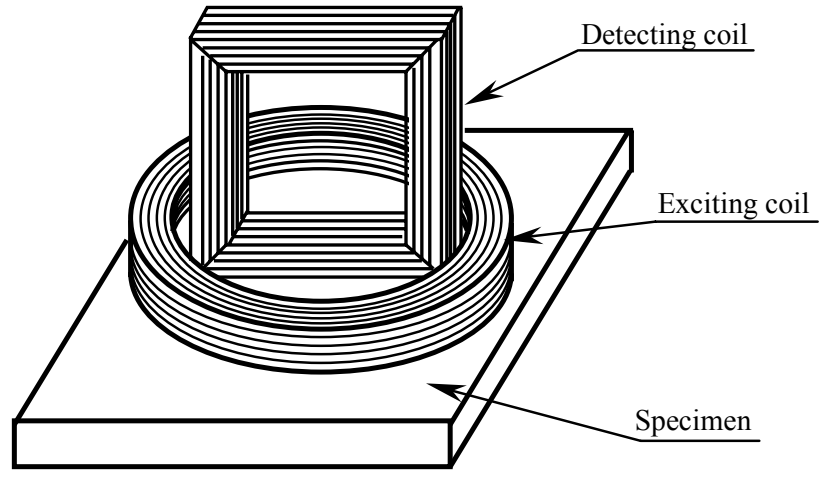

Fig.1 Eddy current testing using the $\Theta$ probe 
When a flaw exists in the specimen, as shown in Fig. 2(a), the electromotive force is generated in the detecting coil as the eddy current flows along the flaw and the component of the eddy current $\mathrm{D}_{\mathrm{A}}$ can be detected. On the other hand, no electromotive force can be detected when the detecting coil is located just on the flaw, as shown in Fig. 2(b), because the eddy current in both sides of the flaw can be mutually cancelled. Moreover, the component of the eddy current $\mathrm{D}_{\mathrm{B}}$ can be detected when the detecting coil passes over the flaw, as shown in Fig. 2(c). Finally, the flaw signal shown in Fig. 2(d) can be obtained in the eddy current testing using the $\Theta$ probe.

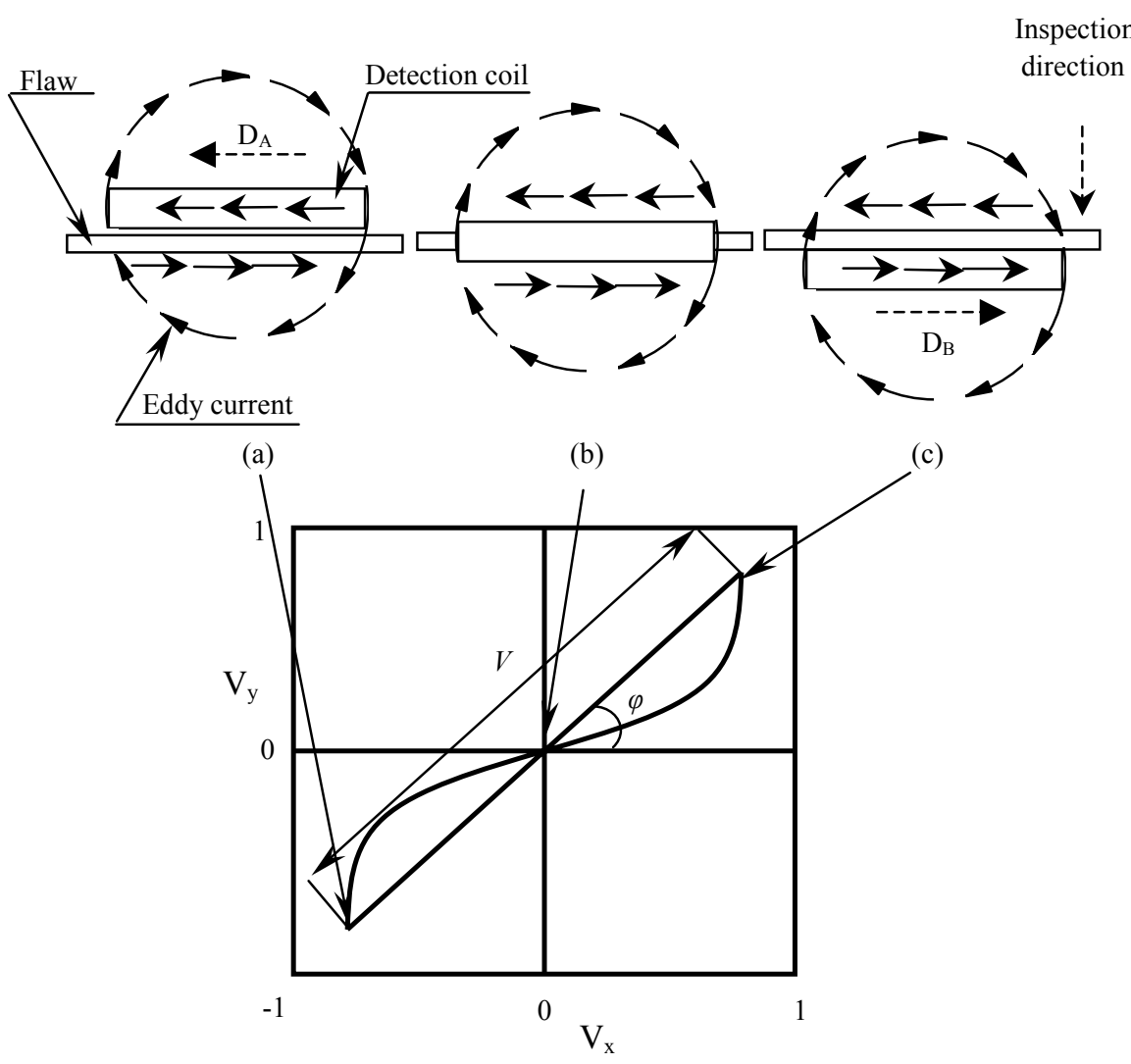

(d) Signal pattern in the eddy current test

Fig.2 Principle of flaw detection by the $\Theta$ probe

\subsection{Caustic Method ${ }^{(6)(7)}$}

The fundamental principle of the caustic method is that the reflected light beam on the surface of a specimen with a crack forms a caustic pattern. The reflected caustics is employed because the specimen material considered in the present study is metal. By measuring the size $D$ of this caustic pattern, the stress intensity factor $K_{I}$ can be determined as follows:

$$
K_{I}=1.671 /\left(z_{0} t c_{0}\right) \cdot\left(1 / \lambda^{1.5}\right) \cdot(D / 3.17)^{2.5}
$$

where $z_{0}$ is the distance between the screen and the specimen, $t$ is the thickness of the specimen, and $\lambda$ is the optical magnification factor. The value $c_{0}$ is an optical constant, which is obtained by the equation $c_{0}=\mu / E$ for reflected caustics. Finally, $\mu$ and $E$ represent Poisson's ratio and Young's modulus, respectively. 


\section{Specimen}

Figure 3 shows the shape of the specimen used to study the fundamental characteristics of the $\Theta$ probe. The material of the specimen is commercially available aluminum plates. An artificial flaw was produced using a high-speed cutter or by electrical discharge machining. The dimensions of the specimen are shown in Table 1. Specimens of various flaw depths $H=0.2 \sim 20 \mathrm{~mm}$, flaw widths $W=0.4 \sim 30 \mathrm{~mm}$, and flaw lengths $L=2 \sim 40$ $\mathrm{mm}$ were prepared in order to test the effect of flaw size. Some of the test specimens in Table 1 were also used to examine experimentally effects such as liftoff.
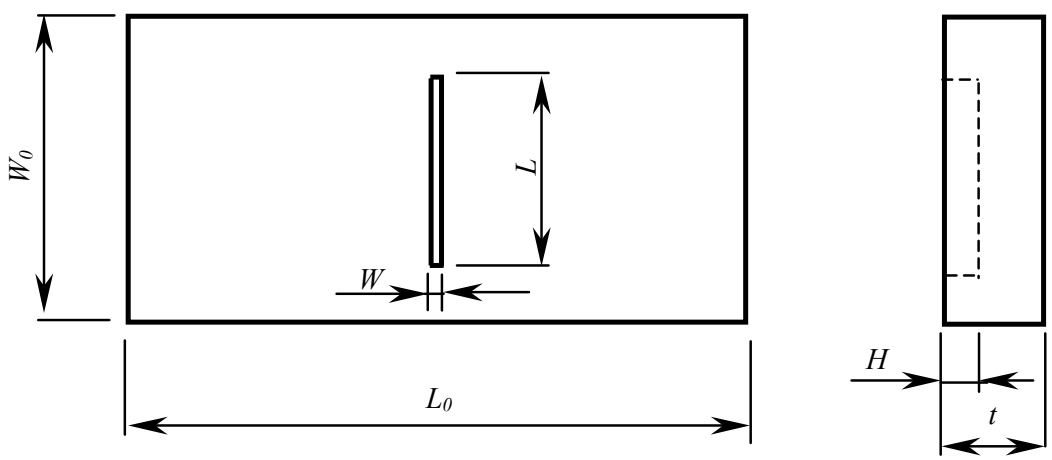

Fig.3 Specimen with an artificial flaw

Table 1 Specimens used to study the fundamental characteristics of the $\Theta$ probe [mm]

\begin{tabular}{|c|c|c|c|c|c|c|}
\hline & \multicolumn{3}{|c|}{ Shape of flaw } & \multicolumn{3}{c|}{ Shape of specimen } \\
\cline { 2 - 7 } & Depth $H$ & Width $W$ & Length $L$ & Width $W_{0}$ & Thickness $t$ & Length $L_{0}$ \\
\hline $\begin{array}{c}\text { Specimen with } \\
\text { various depths }\end{array}$ & $0.2 \sim 20$ & 0.4 & 50 & 50 & 30 & 100 \\
\hline $\begin{array}{c}\text { Specimen with } \\
\text { various widths }\end{array}$ & 0.8 & $0.4 \sim 30$ & 50 & 50 & 10 & 100 \\
\hline $\begin{array}{c}\text { Specimen with } \\
\text { various lengths }\end{array}$ & 0.9 & 0.6 & $2 \sim 40$ & 50 & 10 & 100 \\
\hline
\end{tabular}

Figure 4 illustrates the specimen that was used to attempt the detection of crack initiation under static and repeated loading conditions. In this case, A7075 and A2024 plates, which are widely used as structural materials, were employed. Specimens with an artificial notch of $0.3 \mathrm{~mm}$ in width as well as specimens with natural cracks that were produced in fatigue testing were prepared. The total length $a$ of the crack of the specimen shown in Fig. 4 is approximately $10 \mathrm{~mm}$. The surface of the specimen was highly polished in order to apply the caustic method. The $\Theta$ probe was fixed at the notch tip or at the crack tip, and the specimen was loaded by three-point bending, as shown in Fig. 4.

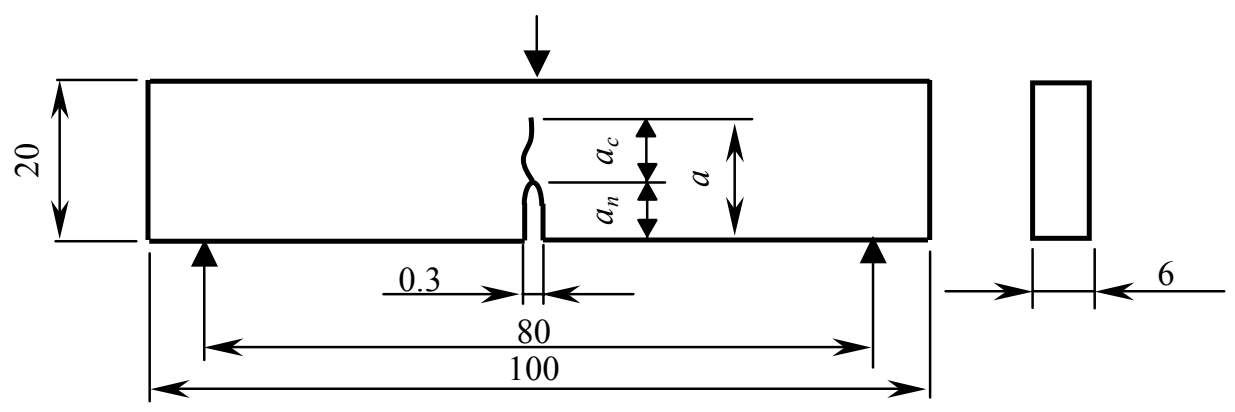

Fig.4 Specimen for the detection of crack initiation under static and repeated loading conditions [mm] 


\section{Experimental Apparatus}

Figure 5 shows the experimental apparatus used to investigate the fundamental characteristics of the $\Theta$ probe. The eddy current testing device ${ }^{(3)}$ is a SUPER TESTER ET-1-2 (Marktec Corp.). In this experiment, a $\Theta$ probe with an outer diameter of $7 \mathrm{~mm}$ (exiting coil diameter: $5 \mathrm{~mm}$ ) is used, and the test is performed by installing the detecting coil essentially parallel to the flaw. A scanning device, by which it is possible to control the probe velocity, is also used. The flaw signal data in the eddy current testing is stored by a personal computer after passing through an oscilloscope. The experimental frequency, amplification, and scanning speed were $25 \mathrm{kHz}, 30 \mathrm{~dB}$, and $0.15 \mathrm{~m} / \mathrm{s}$, respectively.

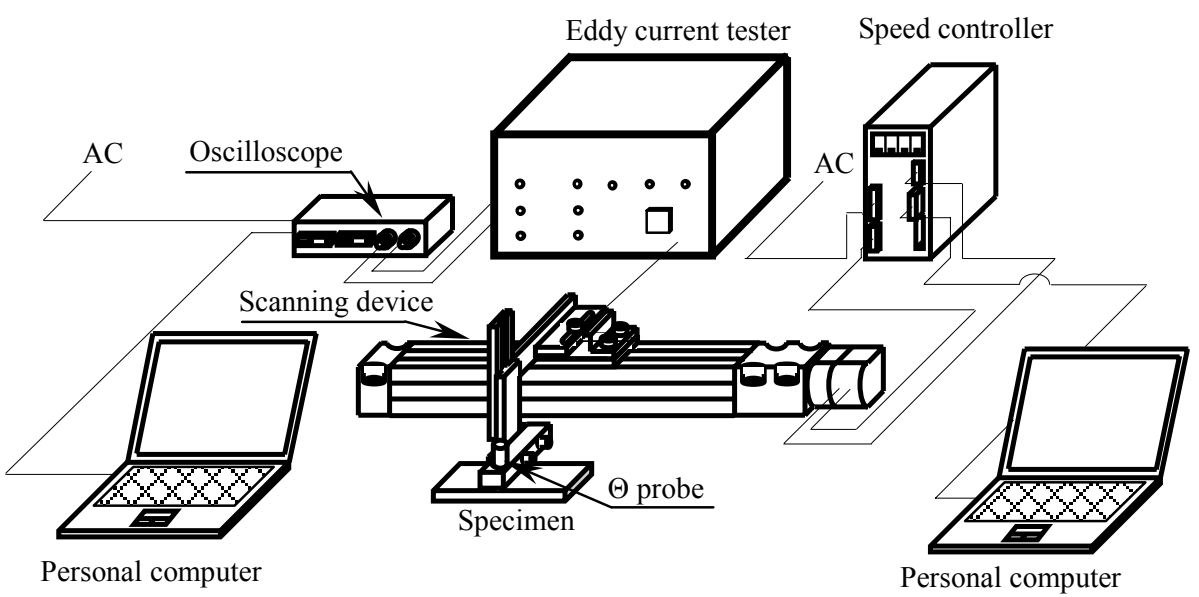

Fig.5 Fundamental apparatus of eddy current test using the $\Theta$ probe

Figure 6 shows the overall experimental setup of the eddy current testing combined with the caustic method. The apparatus used for the eddy current test, shown in Fig. 6, is the same as that shown in Fig. 5, but a $\Theta$ probe with an outer diameter of $11 \mathrm{~mm}$ (exciting coil diameter: $9 \mathrm{~mm}$ ) is employed in order to obtain data over a wide area of the specimen. The $\Theta$ probe was fixed to the specimen. The experiment was carried out under three-point loading using a universal testing machine (AGS-1000B, Shimadzu Co.). The experimental frequency and amplification were $25 \mathrm{kHz}$ and $30 \mathrm{~dB}$, respectively. In the caustic method, the

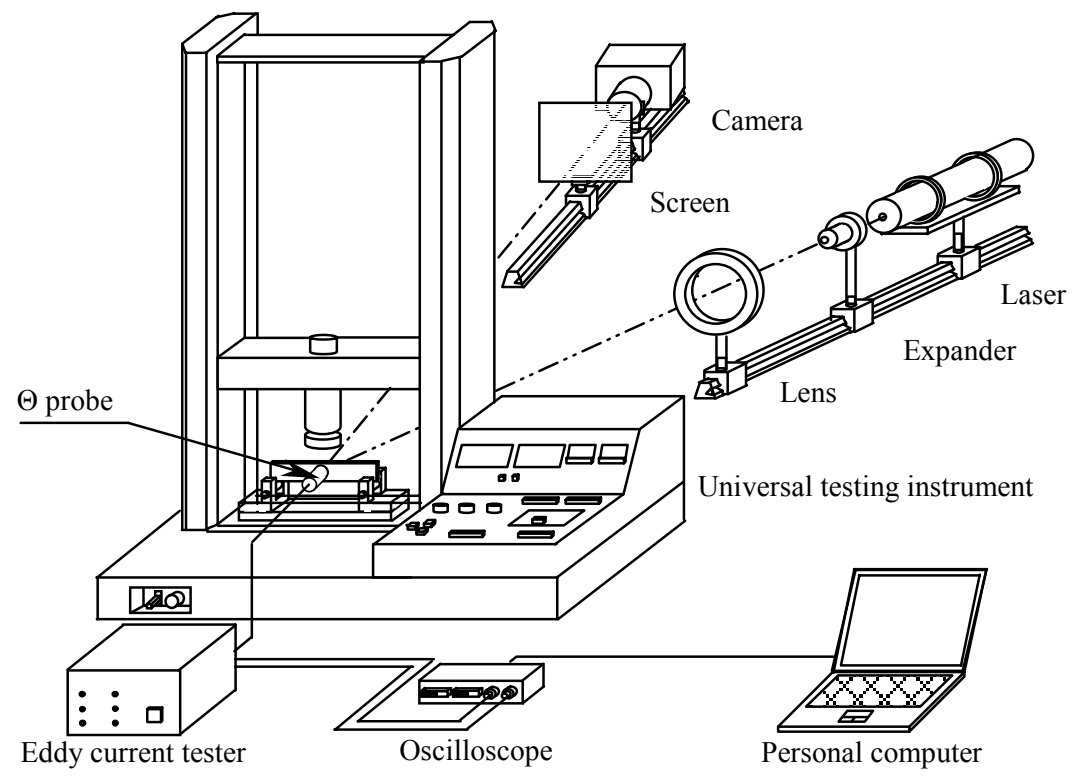

Fig.6 Schematic diagram of the overall experimental setup 
convergent laser light was passed through an expander and the lens from the laser light source. The reflected light on the specimen formed a caustic pattern on the screen, and this pattern was recorded by camera. The distance $z_{i}$ between the specimen and the focal point was $z_{i} \fallingdotseq 400 \sim 500 \mathrm{~mm}$, and the distance $z_{0}$ between the specimen and the screen was $z_{0}$ $\doteqdot 1,600 \sim 2,000 \mathrm{~mm}$. The optical constant $c_{0}$ was $4.7 \times 10^{-12} \mathrm{~m}^{2} / \mathrm{N}$, based on the values of Young's modulus $E=70.6 \mathrm{GPa}$ and Poisson's ratio $\mu=0.33$ of the duralumin plates.

\section{Experimental Results and Discussion}

\subsection{Fundamental Characteristics of the $\Theta$ Probe}

(1) Influence of liftoff

Figure 7 shows the effect of liftoff $y$ on the flaw signal of the $\Theta$ probe. The flaw depth $H$, flaw width $W$, and flaw length $L$ were $H=6.7 \mathrm{~mm}, W=0.4 \mathrm{~mm}$, and $L=50 \mathrm{~mm}$, respectively. Figure 7 shows that the amplitude $V$ is decreased and the phase angle $\varphi$ is delayed as the liftoff $y$ is increased. The experimental results described in Fig. 7 have a similar tendency to those reported previously using brass plates ${ }^{(2)}$. Figure 7 reveals that the $\Theta$ probe enables experimentation at a liftoff of $2 \mathrm{~mm}$ for the aluminum plates and that a liftoff of $3 \mathrm{~mm}$ can be employed if a large amplification is used.

Although the experimental condition is slightly different, the experiment was repeated five times for this test, and results similar to those shown in Fig. 7 were obtained.

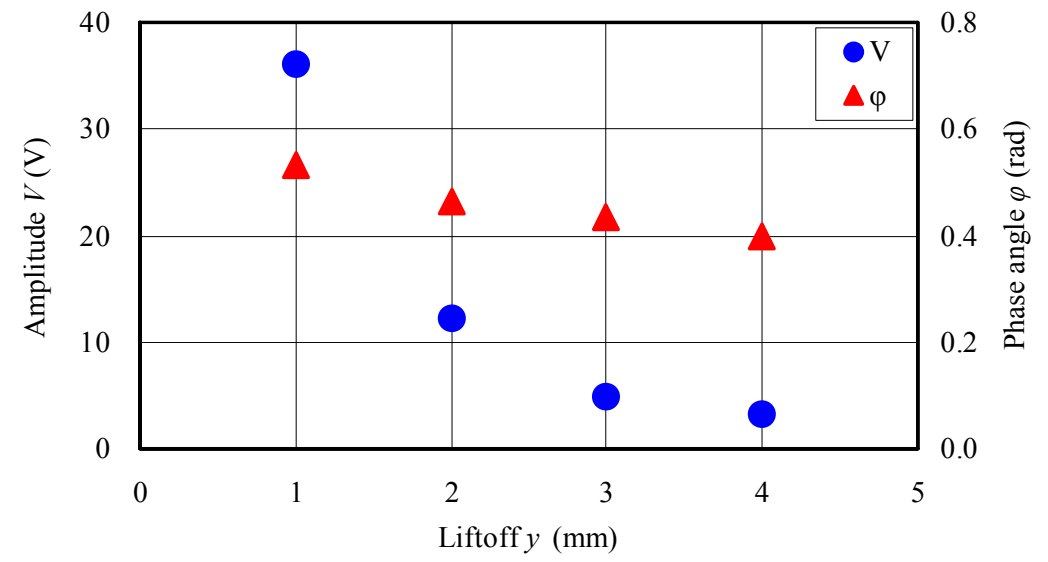

Fig.7 Effect of liftoff $y$ on $V$ and $\varphi$

(2) Effects of the angle between the detecting coil and the flaw

The effects of the angle $\chi$ between the detecting coil and the flaw on the amplitude and the phase angle are analyzed. The angle $\chi$ is defined to be 0 when the detecting coil is positioned parallel to the flaw, as shown in Fig. 8. The liftoff is set to be $1 \mathrm{~mm}$. In this experiment, the flaw depth $\mathrm{H}$ is $2 \mathrm{~mm}$, the flaw width $\mathrm{W}$ is $0.4 \mathrm{~mm}$, and flaw length $\mathrm{L}$ is 50 $\mathrm{mm}$. The experimental results shown in Fig. 9 indicate that the amplitude reaches a maximum at $\chi=0$, where the detecting coil is parallel to the flaw, and reaches a minimum at $\chi=$ $\pi / 2$. Since the component $y$ of the eddy current along the direction of the wound coil, as shown in Fig. 8, can be detected by the $\Theta$ probe, the amplitude is essentially zero at $\chi=$ $\pi / 2$ where the detecting coil is positioned vertical to the flaw. Although a slight variation is observed in the phase angle at $\chi=\pi / 2$, the flaw signal is very small and the effect of the noise of the experimental apparatus becomes relatively large in this case. Therefore, it is considered that no change in the phase angle with respect to $\chi$ will occur.

In this test, the experiment was repeated three times and results similar to those shown in Fig. 9 were obtained. 


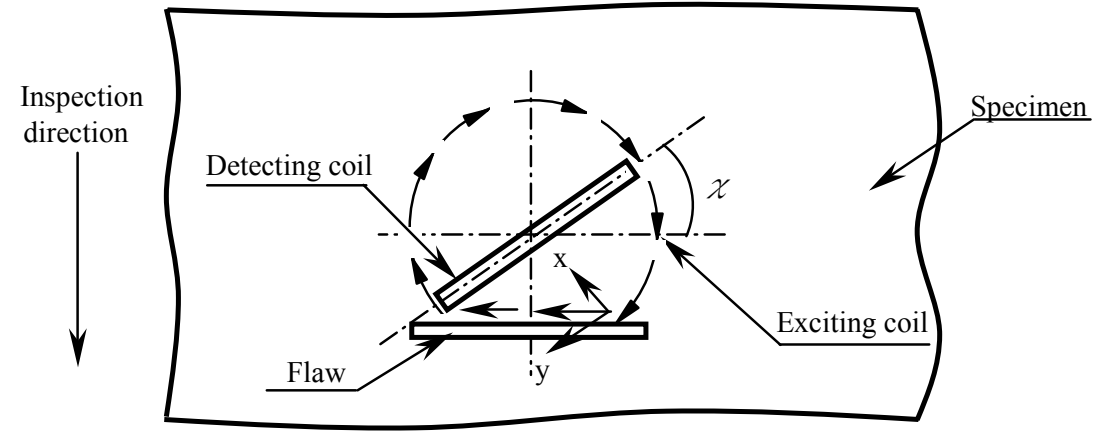

Fig.8 Definition of $x$ in the fundamental test

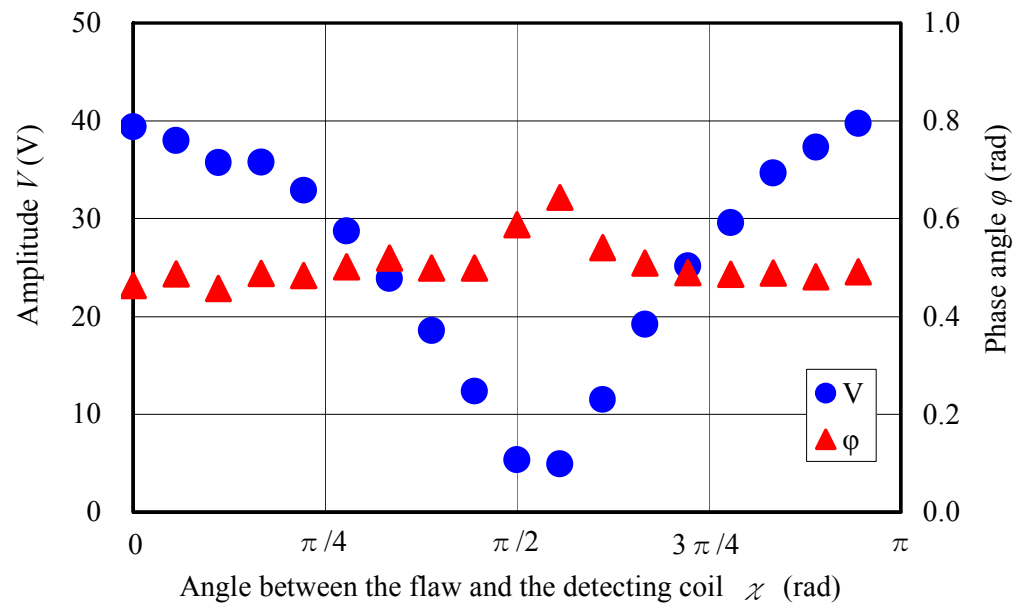

Fig.9 Variations of $V$ and $\varphi$ with respect to $x$

(3) Effects of flaw dimensions

Figure 10 shows the experimental results for detecting an artificial flaw using the $\Theta$ probe at a liftoff of $1 \mathrm{~mm}$. The experimental results denoted by the symbols $H, W$, and $L$ indicate that these results are obtained by changing only the values of the flaw depth, flaw width, and flaw length, respectively, as shown in Table 1. Figure 10 indicates that the amplitude is increased with respective increases in $H, W$, and $L$. The effect of the flaw depth

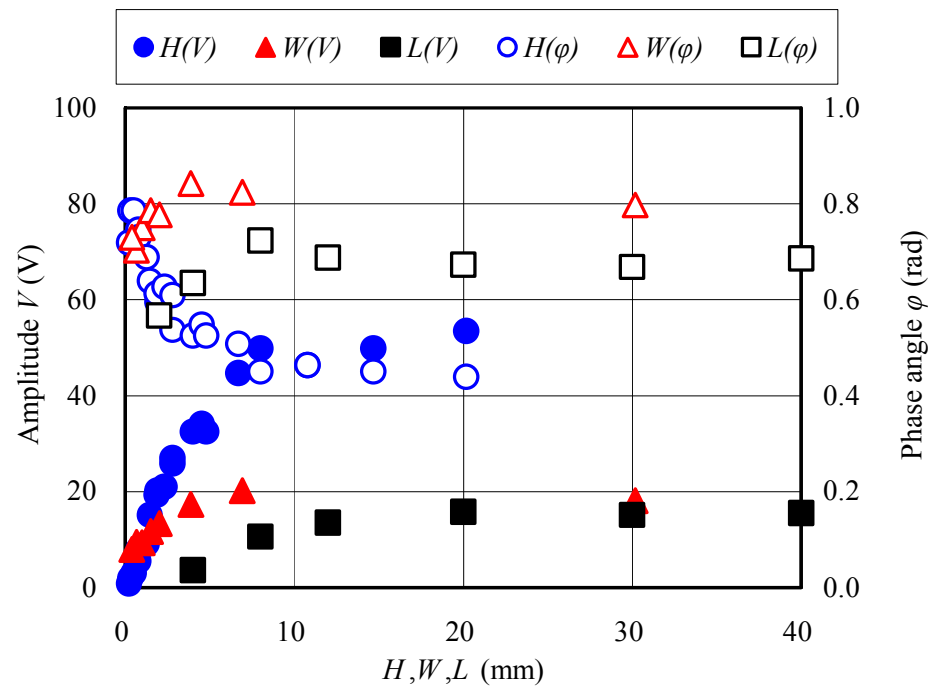

Fig.10 Variations of $V$ and $\varphi$ with respect to flaw dimensions 
$H$ was observed to be large until $H \fallingdotseq 8 \mathrm{~mm}$. The value of $V$ reaches a constant value at $W$ $\fallingdotseq 7 \mathrm{~mm}$ and at $L \fallingdotseq 20 \mathrm{~mm}$. The phase angle $\varphi$ is delayed as the flaw depth is increased and reaches a constant at $H>8 \mathrm{~mm}$. On the other hand, the effects of the flaw width and flaw length on phase angle were not so large.

In this test, we repeated the experiment three times. All of the experimental results showed a tendency similar to that of the data shown in Fig. 10 and yielded approximately the same limiting values of $H, W$, and $L$, which give a constant amplitude.

\subsection{Detection of Crack Initiation Under Static Loading}

(1) Effect of $\eta$ on crack detection sensitivity

Before carrying out the experiment on the detection of crack initiation, the effect of the angle $\eta$ between the detecting coil and the flaw shown in Fig. 11 on the sensitivity of crack detection is investigated. In this test, the $\Theta$ probe is fixed at one point above the notch tip, as shown in Fig. 11. When the crack began to extend at fracture, a change in the eddy current occurs at the crack tip. Therefore, the output voltage can be detected by the $\Theta$ probe. An A7075 plate with an artificial notch of $10 \mathrm{~mm}$ in length, shown in Fig. 4, is used in this investigation. The $\Theta$ probe with a diameter of $11 \mathrm{~mm}$ was positioned at $5.5 \mathrm{~mm}$ upward from the notch tip. In this investigation, the experimental conditions of the liftoff and the amplification were $1 \mathrm{~mm}$ and $20 \mathrm{~dB}$, respectively. In the specimen with the notch, brittle fracture occurred, accompanied by a large amplitude of the $\Theta$ probe.

Figure 12 shows the relationship between $\eta$ and the amplitude of the $\Theta$ probe that was obtained immediately at fracture. This figure shows that the amplitude becomes a minimum at $\eta=0$ and $\pi / 2$ and reaches a maximum at $\eta=\pi / 4$. When the $\Theta$ probe is practically applied to the structural member, it is generally not possible to determine the angle $\eta$ beforehand. Therefore, the angle $\eta=\pi / 2$, which gives a small amplitude, is employed in the following experiments to verify the possibility of applying this technique to practical structures.

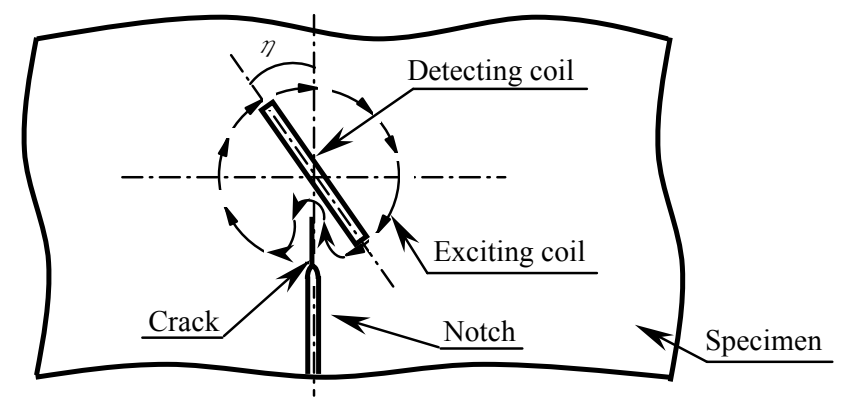

Fig.11 Definition of $\eta$ in the detection test of crack initiation

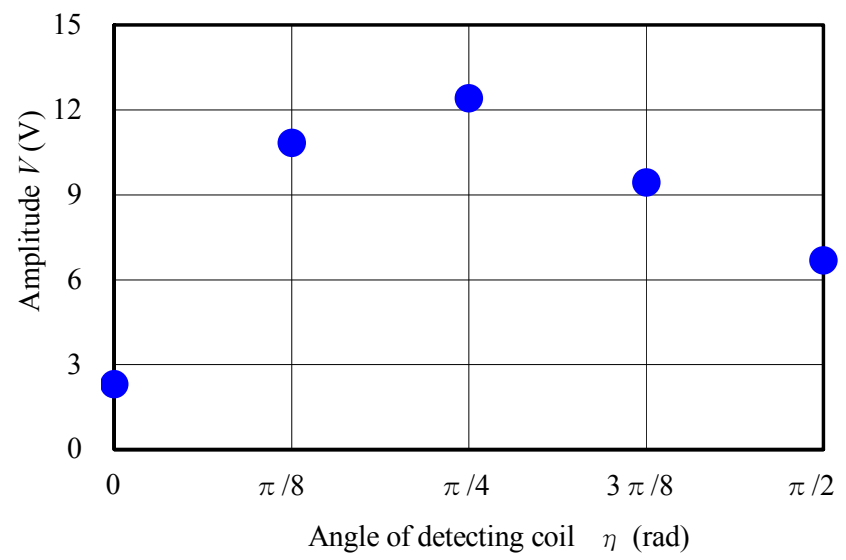

Fig. 12 Variation of $V$ with respect to $\eta$ of the detecting coil 
(2) Crack monitoring under static loading

The detection of fracture initiation is attempted using A7075 duralumin plates under three-point bending, as shown in Fig. 4. The specimen with a natural crack of $7 \mathrm{~mm}$ in length is studied. The $\Theta$ probe with a diameter of $11 \mathrm{~mm}$ is employed, and the experiment is performed by fixing the center of the probe at $5.5 \mathrm{~mm}$ to upward from the crack tip. The probe is installed such that the direction of the detecting coil is at a right angle to the crack line and the liftoff is $1 \mathrm{~mm}$. An example of the obtained caustic pattern is shown in Fig. 13.

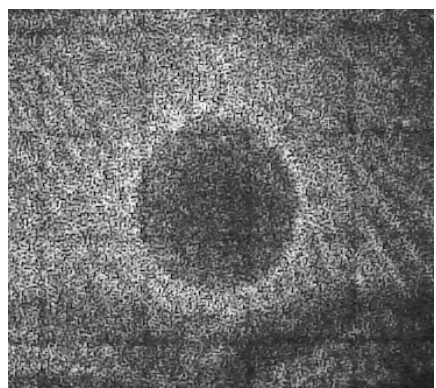

Fig.13 Example caustic pattern (A7075, Crack, $\left.\left(K_{I}\right)_{t h}=24 \mathrm{MPa} \cdot \mathrm{m}^{1 / 2}, z_{i}=500 \mathrm{~mm}, z_{0}=1,600 \mathrm{~mm}\right)$

Figure 14 shows the variations of $\left(K_{I}\right)_{\exp }$ and $\left(K_{I}\right)_{t h}$ with respect to deflection $\delta$. The value of $\left(K_{I}\right)_{\exp }$ is obtained from the caustic pattern, as shown in Fig. 13, and $\left(K_{I}\right)_{t h}$ is calculated from the load $P$. Here, $\left(K_{I}\right)_{t h}$ reaches a maximum of $24 \mathrm{MPa} \cdot \mathrm{m}^{1 / 2}$ at a deflection of $0.6 \mathrm{~mm}$. After this point, a large difference is observed between $\left(K_{I}\right)_{t h}$ and $\left(K_{I}\right)_{\exp }$ due to the plastic deformation. Figures 15(a) 15(d) show the variations in the amplitude of the $\Theta$ probe with time when fracture occurred at each of points A, B, C, and D in Fig. 14. As shown in Fig. 14, in the A7075 plate with a natural crack, fracture occurred intermittently. A small crack extension began at point A accompanied by fracture sound and a slight drop in load. A similar fracture phenomenon was subsequently observed at points B, C, and D with an increase in load, and the total length of the crack extension was $6 \mathrm{~mm}$ at point $\mathrm{D}$. Point $\mathrm{A}$ indicates the beginning stage of fracture in the elastic range, and a small amplitude of the $\Theta$ probe is observed due to fracture. On the other hand, at point $\mathrm{D}$, plastic deformation

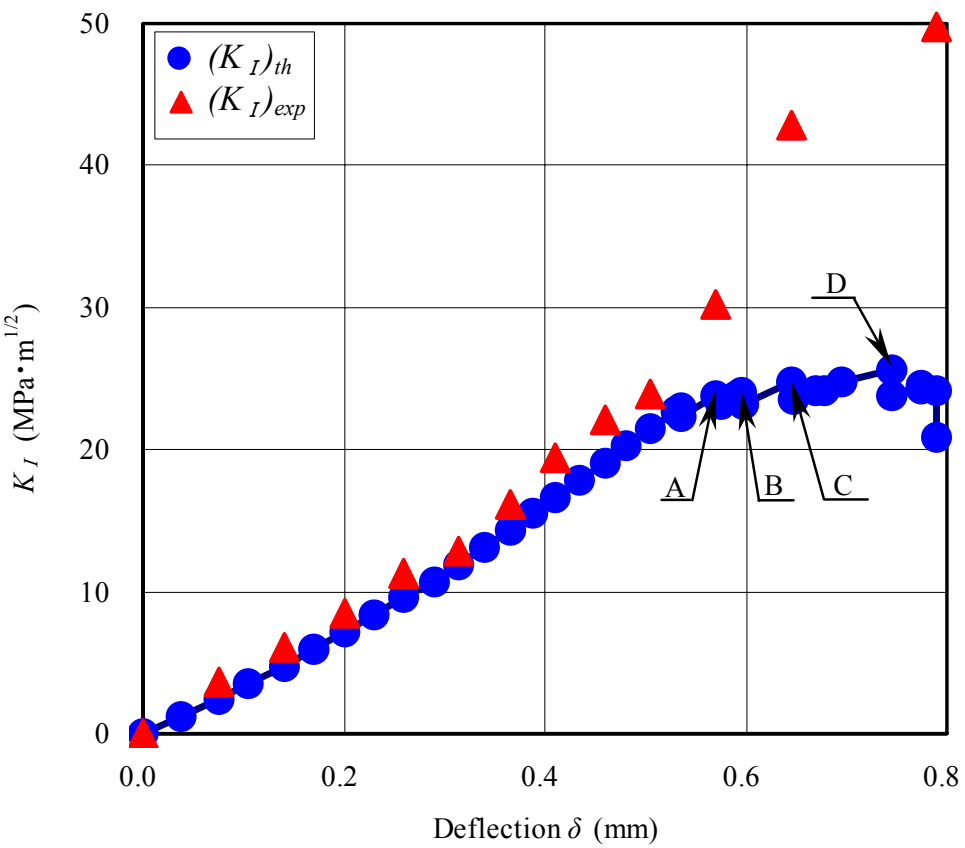

Fig.14 Variation of $\mathrm{K}_{\mathrm{I}}$ with respect to $\delta$ (A7075, Crack) 
is observed in the specimen, and a large amplitude is observed. In addition, a change in the sign of the amplitude is observed at points $\mathrm{C}$ and $\mathrm{D}$. This is considered to be caused by the change in the mutual positions of the crack tip and the $\Theta$ probe, which is induced by the crack extension. As mentioned above, it is possible to detect the initiation of a crack under static loading using the $\Theta$ probe. The experimental result in Fig. 15 is obtained by setting a high pass filter (HPF) at $5 \mathrm{~Hz}$. Although it is not possible to remove the HPF from the eddy current test equipment used in this experiment, if it were possible, then some change in the variation of the amplitude in Fig. 15 is expected to occur. We intend to investigate this point in the future.

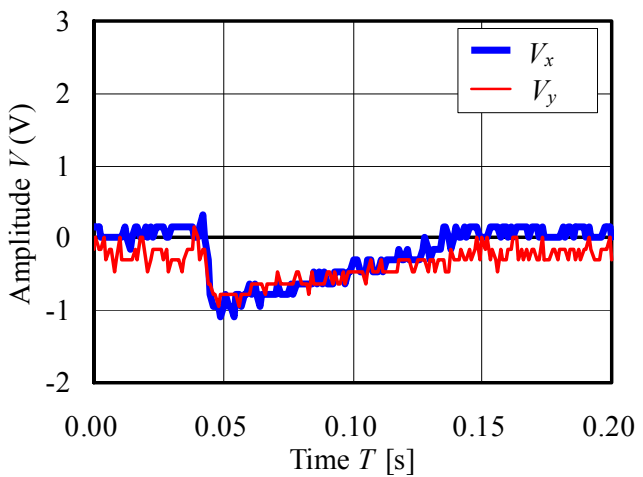

(a)Point $\mathrm{A}(\delta=0.53 \mathrm{~mm})$

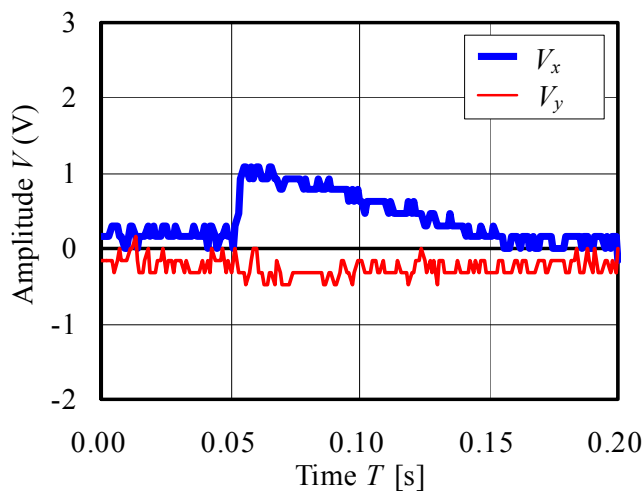

(c)Point $\mathrm{C}(\delta=0.65 \mathrm{~mm})$

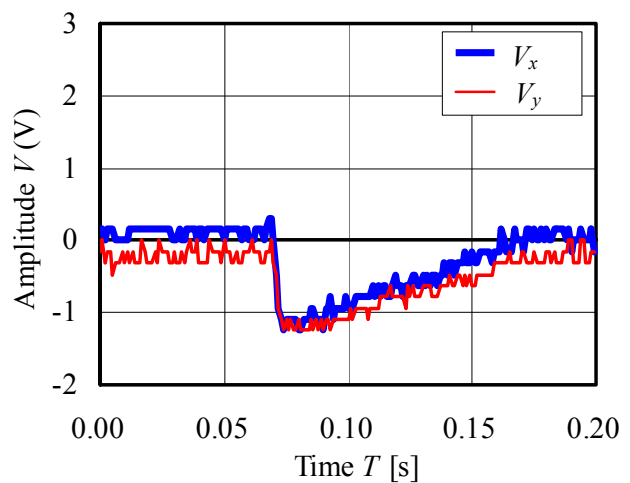

(b)Point $\mathrm{B}(\delta=0.57 \mathrm{~mm})$

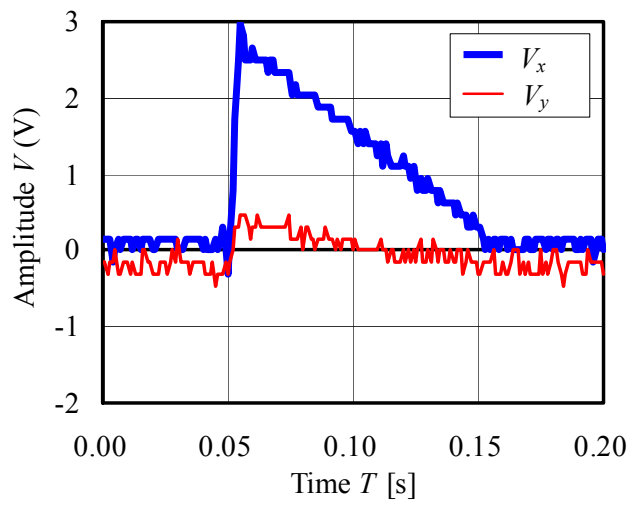

(d)Point $\mathrm{D}(\delta=0.77 \mathrm{~mm})$

Fig.15 Variation of amplitude with respect to time at fracture (A7075, Crack)

Crack monitoring of the A2024 plate, which indicates more ductile fracture behavior compared to A7075, is also investigated. The experimental conditions are the same as those used for the A7075 plate, and no amplitude was observed in this case. Figure 16 shows the experimental result obtained using a liftoff of $0 \mathrm{~mm}$ for the specimen with a notch. Although the conditions by which a large amplitude of the $\Theta$ probe can be obtained are unique, there is no definite change in the amplitude, as shown in this figure. Consequently, it is considered that crack monitoring is impossible in the A2024 plate.

The value of $\mathrm{S} / \mathrm{N}$ is approximately $2 \sim 6$ for the experimental results of $V_{x}$ shown in Fig. 15. Experiments concerning the results in Figs. 15 and 16 were carried out using two test specimens in each case, and results similar to those in Figs. 15 and 16 were obtained. This confirms that the experimental results shown in Fig. 15 indicate the possibility of crack monitoring in the A7075 plate.

In the A2024 plate, which is more ductile than the A7075 plate, as mentioned above, fracture occurred without fracture sound or a slight drop in load, which were observed in 
the A7075 plate, and the crack extended slowly. Such a difference in the fracture behavior is considered to be the reason why crack monitoring is possible in the A7075 plate, but is not possible in the A2024 plate.

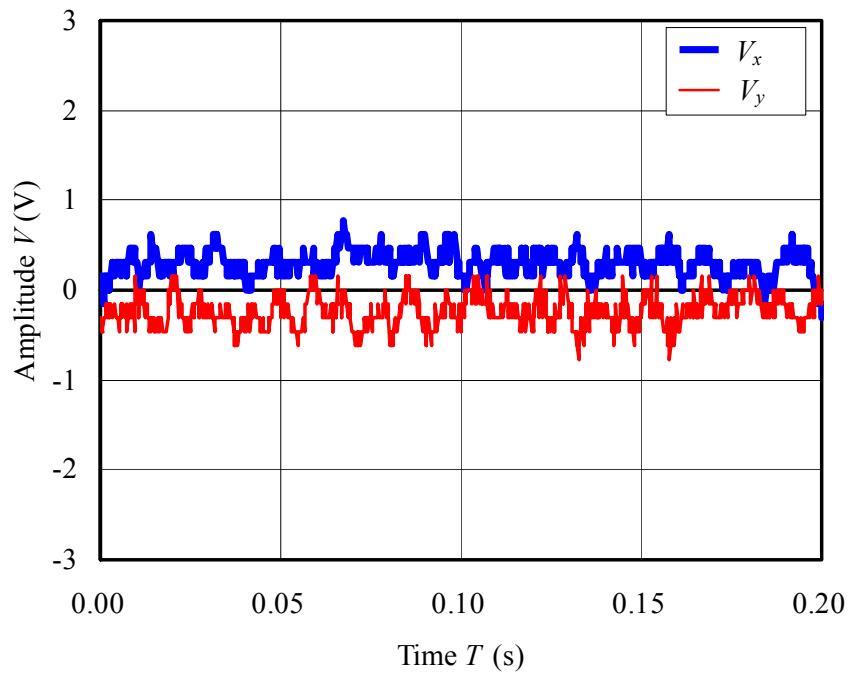

Fig.16 Variation of amplitude with respect to time at fracture (A2024, Notch)

\subsection{Detection of Crack Growth Under Low Cycle Fatigue Condition}

The amplitude of the $\Theta$ probe is measured when the crack began to extend from a notch under the repeated loading condition. The $\Theta$ probe of $11 \mathrm{~mm}$ in diameter was fixed with its center at $5.5 \mathrm{~mm}$ upward from the notch tip. The probe is installed such that the direction of the detecting coil is at a right angle to the crack line and the liftoff is $1 \mathrm{~mm}$. Crack length is measured using an optical microscope with a magnification factor of 30 . A low cycle fatigue test was performed at a stress ratio $R$ of 0 under three-point loading. The testing machine is arranged such that the initial stress intensity factor range was $\Delta K \fallingdotseq 20 \mathrm{MPa} \cdot \mathrm{m}^{1 / 2}$, and the experiment was performed at a constant displacement.

The experimental results for the A7075 plate are shown in Fig. 17, which shows the effects of the fatigue crack growth rate $d a / d N$ on the amplitude $V$ and the phase angle $\varphi$. In this figure, the values of $\left(\Delta K_{I}\right)_{t h}$ and $\left(\Delta K_{I}\right)_{\exp }$ are also illustrated to show the deformation

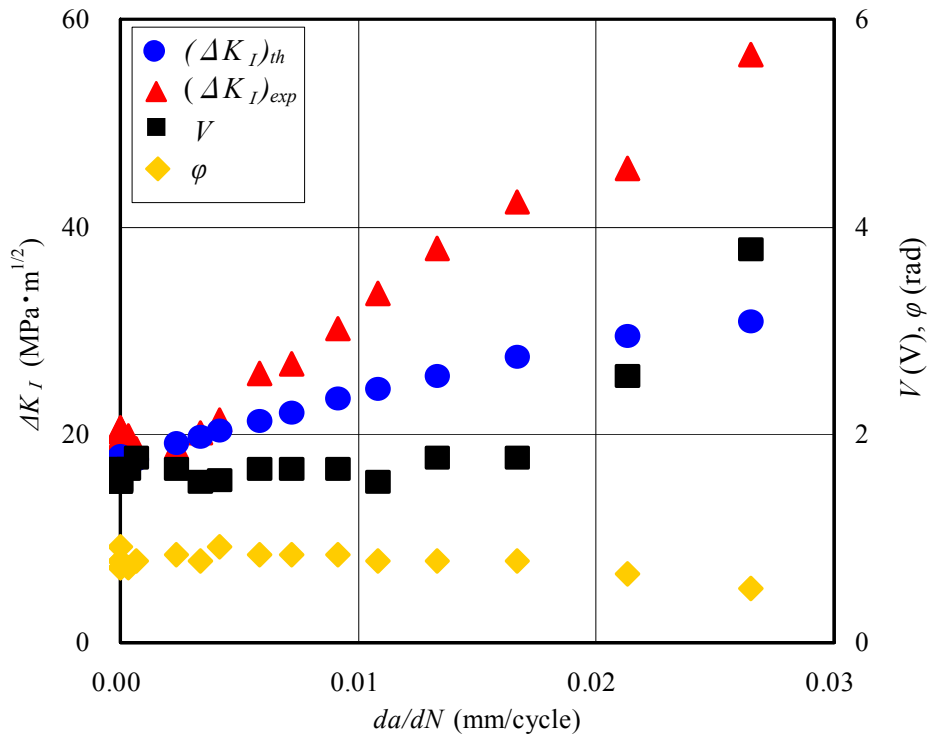

Fig. 17 Variations of $\Delta K_{I}, V$, and $\varphi$ with $d a / d N$ under fatigue testing (A7075) 
state in the vicinity of the crack tip. Although the values of $\left(\Delta K_{I}\right)_{t h}$ and $\left(\Delta K_{I}\right)_{\text {exp }}$ coincide in the initial stage, $\left(\Delta K_{I}\right)_{\exp }$ becomes larger than $\left(\Delta K_{I}\right)_{t h}$ as deformation proceeds, which means that plastic deformation occurred at the crack tip. The results shown in this figure indicate that the amplitude $V$ is increased and the phase angle is delayed when the value of $d a / d N$ becomes larger than $0.017 \mathrm{~mm} /$ cycle. Thus, it is verified that fatigue crack monitoring is possible using the $\Theta$ probe for large fatigue crack growth rates. It is considered that fatigue crack monitoring by the $\Theta$ probe will be possible even at smaller value of $d a / d N$ if the experiment is carried out with small liftoff and large amplification.

In this test, an experiment was carried out using three test specimens, and results similar to those shown in Fig. 17 were obtained. Consequently, the reproducibility of the results shown in Fig. 17 is considered to be fairly good.

\section{Conclusions}

The fundamental characteristics of the $\Theta$ probe were investigated using commercially available aluminum plates. In addition, the possibility of detecting crack initiation in duralumin plates under static and low cycle fatigue conditions was investigated by applying the $\Theta$ probe combined with the caustic method. The experimental results obtained under the experimental conditions of the present study are as follows:

(1) The effects of liftoff and the angle between the detecting coil and the flaw on the amplitude and the phase angle of the $\Theta$ probe were clarified.

(2) The effects of the flaw dimensions on the flaw signal were determined. The amplitudes of specimens with various flaw depths $H$ increased greatly to $H \doteqdot 8 \mathrm{~mm}$. The amplitude reached a constant value for the specimen with flaw width $W$ of more than 7 $\mathrm{mm}$ and for the specimen with the flaw length $L$ of more than $20 \mathrm{~mm}$.

(3) The effect of $\eta$, the angle between the detecting coil and the flaw, on the amplitude at initiation of fracture in A7075 plate with a notch under static loading was analyzed, and the amplitude was shown to reach a maximum at $\eta=\pi / 4$.

(4) A large amplitude could be obtained at crack initiation for the A7075 plate with a natural crack by setting $\eta=\pi / 2$ under static loading over a wide range of deformation states in the vicinity of the crack tip. Crack monitoring in the A7075 plate using the $\Theta$ probe was verified to be possible. However, crack monitoring was not possible in the A2024 plate.

(5) The amplitude could be observed at a fatigue crack growth rate greater than 0.017 $\mathrm{mm} /$ cycle by applying the $\Theta$ probe on the A7075 plate, and the possibility of fatigue crack monitoring using the $\Theta$ probe was confirmed.

\section{Acknowledgements}

The authors would like to thank Dr. Hiroshi Hoshikawa and Dr. Kiyoshi Koyama of Nihon University for their helpful comments and suggestions regarding the $\Theta$ probe.

\section{References}

(1) Hoshikawa, H., Koyama, K. and Karasawa, H., Study of a New Surface Eddy Current Probe without Lift-off Noise (in Japanese), Journal of the Japanese Society for Non-Destructive Inspection, Vol.50, No.11(2001), pp.736-742.

(2) Hoshikawa, H. and Koyama, K., A New Eddy Current Probe with Minimal Liftoff Noise and Phase Information on Discontinuity Depth, Materials Evaluation, Vol.61, No.3(2003), pp.423-427.

(3) Hiroshima, T., Detection of a Crack by Theta Probe (in Japanese), Journal of the Japanese 
Society for Non-Destructive Inspection, Vol.52, No.11 (2003), pp.605-609.

(4) Fukutomi, H., Nomoto, A. and Ogata, T., Accurate Crack Sizing of Land-Based Gas Turbine Blades by Eddy-Current Techniques (in Japanese) Transactions of the Japan Society of Mechanical Engineers, Series A, Vol.69, No.685(2003), pp.1337-1344.

(5) Kasuya, T., Endo, H., Uchimoto, T. and Takagi, T., Quantitative Evaluation of Crack Propagation Based on in-situ Eddy Current Monitoring System (in Japanese) Transactions of the Japan Society of Mechanical Engineers, Series A, Vol.72,No.714(2006), pp.165-172.

(6) Shimizu, K. and Suetsugu, M., Photomechanics (Takahashi, S. ed.) (in Japanese), (1997), pp.129-156, Sankaido.

(7) Kalthoff, J.F., Handbook on Experimental Mechanics (Kobayashi, A. S. ed.), (1987), p.430, Prentice-Hall, Inc. 